\title{
Cardiomiopatia de cães da raça Cocker Spaniel Inglês: aspectos clínicos, eletrocardiográficos, radiográficos e ecocardiográficos ${ }^{1}$
}

\author{
Cardiomyopathy of the English Cocker Spaniel: clinical, electrocardiographic, \\ radiographic and echocardiographic aspect
}

\author{
Luciano Pereira ${ }^{2}$ Maria Helena Matiko Akao Larsson ${ }^{3}$ \\ Moacir Leomil Neto ${ }^{4}$ Fábio Sandoli de Brito ${ }^{5}$
}

\section{RESUMO}

Face aos poucos relatos sobre a cardiomiopatia na raça Cocker Spaniel Inglês na literatura compilada, o presente trabalho visou contribuir para melhor elucidação das lacunas relativas ao seu diagnóstico, e somar conhecimentos para sua caracterização com relação aos aspectos clínicos, eletrocardiográficos, radiográficos e ecocardiográficos. Os estudos foram conduzidos em vinte animais da raça Cocker Spaniel Inglês, portadores da cardiomiopatia dilatada e submetidos à avaliação clínica, eletrocardiográfica, radiográfica, ecocardiográfica, exames laboratoriais e determinação da pressão arterial. $O$ tratamento foi individualizado na dependência da gravidade da insuficiência cardíaca congestiva. Os resultados obtidos confirmaram o longo período assintomático da doença, sendo a insuficiência cardíaca congestiva o evento terminal, em decorrência da perda continuada da força contrátil do miocárdio. A idade média dos animais acometidos foi de sete anos. A sintomatologia foi diretamente relacionada com a diminuição do débito cardíaco. A maioria dos animais apresentou uma evolução clínica favorável da insuficiência cardíaca congestiva; todavia, naqueles com severa cardiectasia ao exame radiográfico, surgimento abrupto de taquiarritmias atriais e redução acentuada da função contrátil ao ecocardiograma, observou-se a ocorrência de uma insuficiência cardíaca congestiva esquerda e também direita, refratárias ao protocolo terapêutico empregado. A eletrocardiografia representou o exame subsidiário de maior diferenciação em relação ao padrão congestivo das raças grandes e gigantes. A maioria dos animais revelou variações do ritmo sinusal, sendo as arritmias supraventriculares observadas nos animais com aumentos importantes das câmaras atriais, constituindo, ainda, aqueles de prognóstico mais reservado. $O$ aumento da amplitude da onda $R$, a maior duração do complexo QRS e a maior profundidade da onda
$Q$, na derivação $I I$, constituíram um bom indicador para o seu diagnóstico. Uma correlação positiva entre os achados eletrocardiográficos e ecocardiográficos foi observada somente em relação à duração do complexo QRS. A função ventricular apresentou valores médios de $19 \%$, sendo observados índices inferiores a $12 \%$ em cinco animais, indicativo de severa disfunção contrátil. Não obstante, a longa sobrevida de um destes animais indicou a baixa confiabilidade dos índices de ejeção como fator único na determinação de um prognóstico.

Palavras-chave: Cocker Spaniel Inglês, coração, cardiomiopatia dilatada.

\section{ABSTRACT}

Since there are few reports on the cardiomyopathy in the English Cocker Spaniel on literature, the present study intends to contribute for the better characterization of its diagnosis as well as to add knowledge for its clinical, electrocardiographic, radiographic and echocardiographic aspects. Twenty Cocker Spaniel dogs with dilated cardiomyopathy were evaluated for electrocardiography, radiography, echocardiography, biochemical serum profiles and arterial pressure determination. The treatment was individualized in the dependence of the gravity of the congestive heart failure. The results confirmed the long assintomatic period of the disease, being the congestive heart failure the terminal event in its evolution due to the continued loss of the myocardial contractile force. The average age of the animals was seven years old. The clinical manifestations were directly related to decrease of the cardiac output. Most of the animals presented a favorable clinical evolution of the congestive heart failure, although, the occurrence of the disease refractory to the therapeutic protocol was identified in those animals with severe biventricular enlargement in the radiographic exam,

${ }^{1}$ Faculdade de Medicina Veterinária e Zootecnia (FMVZ), Universidade de São Paulo (USP). - apoio financeiro: FAPESP

${ }^{2}$ Médico Veterinário, Professor, Mestre, UNI-ABC e Anhembi-Morumbi.

${ }^{3}$ Médico Veterinário, Professora Associada, FMVZ, USP

${ }^{4}$ Médico Veterinário, Professor, Mestre, PUC-MG e FEOB,Rua Caminho Cinco n.20, 37704-403, Bortolan, Poços de Caldas,

MG. E-mail: mleomil@pucpcaldas.br Autor para correspondência.

${ }^{5}$ Médico, Intituto Prócordis de São Paulo. 
abrupt appearance of atrial tachyarrhythmias and accentuated reduction of the contractile function in the echocardiography. The electrocardiography represented the best exam to differentiate the cardiomyopathy of the large breeds from the medium ones. Most of the animals revealed variations of the sinusal rhythm, being the supraventricular arrhythmias observed in the animals with important increases of the atrial chambers, representing those of poor prognostic. The increase on the width of $R$ wave, the largest duration of QRS complex and the largest depth of the $Q$ wave, in the derivation II, constituted good indicators for its diagnosis. A positive correlation between the electrocardiographic and echocardiographic results was only observed in relation to the duration of $Q R S$ complex. The ventricular function presented mean values of 19\%, and lower values (less than $12 \%$ ) were observed in five animals, showing a severe contractive disfunction. Nevertheless, the long survival of one of these animals indicated the low reliability of the ejection fraction index as the most important factor in determining of the prognosis.

Key words: Cocker Spaniel Inglês, heart, dilated cardiomyopathy.

\section{INTRODUÇÃO}

O termo cardiomiopatia (CM) tem sido usado para representar as várias formas de disfunção miocárdica, caracterizadas pela redução da contratilidade e/ou alteração do preenchimento ventricular, e/ou arritmias cardíacas, em ausência de doença valvar ou de malformação congênita (GOODWIN, 1973; LARSSON et al., 1996). A CM dilatada de natureza idiopática refere-se às doenças originadas no miocárdio, cuja etiologia ainda permanece desconhecida e com prognóstico variando de reservado a grave (ATKINS \& SNYDER,1991; KRAMER, 1995).

Esta forma de cardiomiopatia caracterizase pela diminuição da contratilidade, e pelo aumento dos volumes sistólico e diastólico ventriculares (GOODWIN, 1973; BOON, 1998). Ela afeta, predominantemente, cães machos, de raças grandes e gigantes, particularmente o Doberman, Boxer, Dog Alemão, Irish Wolfhound e São Bernardo (HILL, 1981; WARE \& BONAGURA, 1986; ATKINS \& SNYDER,1991; BUCHANAN, 1992), apresentando como manifestações clínicas mais consistentes: astenia, anorexia, letargia, dispnéia, intolerância ao exercício, perda de peso, ascite e síncope (HILL, 1981; WARE \& BONAGURA, 1986; SISSON \& THOMAS,1995). A sua evolução, relativamente benigna, com um longo período assintomático é um dos pontos irrefutáveis que caracterizam a cardiomiopatia, em sua forma dilatada, nos cães da raça Cockers Spaniel Inglês (FOX, 1988; KITTLESON, 1998).
Não obstante, apesar de descoberta há praticamente duas décadas, a cardiomiopatia dilatada do Cocker Spaniel Inglês apresenta uma notória escassez de trabalhos em relação à evolução clínca da doença. Portanto o objetivo do presente estudo é determinar as principais características clínicas, eletrocardiográficas, radiográficas e ecocardiográficas de cães da raça Cocker Spaniel Inglês com cardiomiopatia dilatada e, possivelmente, contrubuir com o conhecimento de sua etiologia.

\section{MATERIAL E MÉTODOS}

Foram utilizados 20 cães, da raça Cocker Spaniel Inglês, de ambos os sexos, com idades diversas, com diagnóstico de cardiomiopatia do tipo dilatada, selecionados pelo então Serviço de Eletrocardiografia (atualmente, Serviço de Cardiologia) do Hospital Veterinário da Universidade de São Paulo.

Os animais foram avaliados por meio da anamnese, exames físico, radiográfico, eletrocardiográfico, ecocardiográfico, avaliação de pressão arterial, exames laboratoriais (funções renal atraves da dosagem plasmática de uréia e creatinina e função hepática através da dosagem plasmática de aspartato aminotransferase, alanina aminotransferase e fosfatase alcalina, hemograma, glicemia e pesquisa de microfilárias circulantes) e avaliação dinâmica do eletrocardiograma por 24horas.

No tocante ao tratamento, os animais foram submetidos a uma conduta terapêutica individualizada, baseada no controle da insuficiência cardíaca congestiva, de caráter emergencial ou crônico. Nos casos agudos (edema pulmonar, efusões pleurais, ascite com comprometimento da função respiratória), a terapêutica instituída variou no uso combinado ou isolado de venodilatador (nitroglicerina ${ }^{a}$ ), broncodilatador (aminofilina ${ }^{\mathrm{b}}$ ), diurético (furosemida ${ }^{\mathrm{c}}$ ), oxigenoterapia, bem como de drenagem de coleções pleurais e/ou abdominais. Com relação ao controle crônico, este dependeu do grau de insuficiência cardíaca congestiva, variando desde o emprego da restrição de sódio alimentar nas fases iniciais, até o uso de diuréticos (furosemida ${ }^{\mathrm{d}}$ e/ou espironolactona ${ }^{\mathrm{e}}$ ), broncodilatadores (aminofilina ${ }^{\mathrm{f}}$ ou teofilina ${ }^{\mathrm{g}}$ ), vasodilatador (maleato de enalapril ${ }^{\mathrm{h}}$ ) e digitálico (digoxina $^{\mathrm{i}}$ ), de forma isolada ou em associações terapêuticas nos estágios mais severos. Deve-se ressaltar que, em relação à restrição de sódio alimentar, esta foi incluída como parte do protocolo de tratamento, somente naqueles animais que receberam, por, pelo menos algumas semanas, as rações comerciais para cardiopatas $\left(\mathrm{CV}^{\circledR}\right.$ - Purina ou K/D Hill's $\left.{ }^{\circledR}\right)$, não sendo, 
portanto, considerados os animais com alimentação exclusiva por meio de dieta caseira hipossódica. $\mathrm{Na}$ presença de outros quadros mórbidos concomitantes à insuficiência cardíaca congestiva, a conduta terapêutica adotada foi prescrita individualmente em cada caso clínico.

Os resultados eletrocardiográficos de amplitude de onda R, duração do complexo QRS e amplitude da onda Q, na derivação II, dos animais com cardiomiopatia dilatada, foram submetidos à análise estatística pelo método de regressão linear, com os dados de função ventricular obtidos, ecocardiograficamente (SPIEGEL,1972).

\section{RESULTADOS E DISCUSSÃO}

A idade média dos animais acometidos foi de sete anos, contudo o presente trabalho identificou um animal com apenas sete meses de idade com severa dilatação cardíaca, acompanhada por edema pulmonar. Não se observou uma predisposição sexual, já que a porcentagem de machos acometidos (10 animais) foi igual à das fêmeas.

A sintomatologia nos animais foi predominantemente relacionada à diminuição do débito cardíaco, e conseqüente intolerância à atividade física, tosse improdutiva, essa sendo resultado da compressão do brônquio esquerdo principal, em decorrência ao aumento da câmara atrial esquerda.

Quando da evolução para a insuficiência cardíaca congestiva, a forma predominante foi a esquerda (18 animais). Porém, em cinco animais com cardiectasia grave e perda significativa da função contrátil do miocárdio, observou-se à presença concomitante de um quadro congestivo direito, representado por efusão abdominal (ascite).

A evolução lenta e progressiva da doença até o surgimento de manifestações clínicas de insuficiência cardíaca congestiva foi observada, claramente, em seis animais, mas cinco deles manifestaram surgimento repentino dos sintomas de insuficiência cardíaca congestiva, sem qualquer manifestação prévia de uma doença cardíaca em evolução.

Dos 20 animais avaliados, 18 mostraram sinais clínicos de insuficiência cardíaca congestiva; destes, oito apresentaram uma evolução clínica favorável, já que os episódios congestivos agudos com o protocolo terapêutico instituído foram controlados satisfatoriamente. O óbito não raramente foi causado por outras doenças que não a cardiomiopatia, como a insuficiência renal, observada em dois deles. Neste aspecto, dois animais merecem um destaque especial, um pela longa sobrevida desde o início dos sintomas (5 anos) e o outro pela manutenção de um quadro clínico estável, mesmo durante e após uma prenhez, não recomendada.

Apesar da resposta satisfatória dos cães da raça Cocker Spaniel Ingles ao tratamento para a insuficiência cardíaca congestiva, foi possível evidenciar no presente estudo três animais que desviaram deste padrão. Neles, foi evidente uma evolução extremamente rápida da insuficiência cardíaca congestiva esquerda (um animal) e também direita (dois animais), ambos refratários ao protocolo terapêutico empregado, determinando o óbito dos mesmos, poucas semanas após o início dos sintomas.

Os achados radiográficos revelaram, de forma geral, o aumento global da silhueta cardíaca com elevação dorsal da traquéia. Nos casos mais graves, a presença de congestão dos vasos pulmonares ou mesmo de edema intersticial ou alveolar foram indicativos da presença de insuficiência cardíaca congestiva esquerda.

Eletrocardiograficamente, a literatura relata que ondas $\mathrm{R}$ com amplitude maior que $2,5 \mathrm{mV}$, na derivação II, por vezes associado a um "slurring" do segmento ST, representam um grande indicador da dilatação ventricular esquerda. Neste particular, 11 dos 20 animais acometidos corroboram a relação entre amplitude de onda $\mathrm{R}$ e tamanho de câmara cardíaca pois apresentaram amplitudes da onda $\mathrm{R}$ maior $2,5 \mathrm{mV}$, somados a uma severa dilatação cardíaca, ao exame radiográfico, e um aumento da cavidade ventricular esquerda, ao exame ecocardiográfico. Um dos animais, com ondas $\mathrm{R}$ de até $6,5 \mathrm{mV}$ e "slurring" do segmento ST, representou um bom exemplo desta relação ao evidenciar a cardiectasia, com desvio dorsal da traquéia ao exame radiográfico, bem como o aumento severo da cavidade ventricular esquerda $(5,3 \mathrm{~cm})$, e conseqüente diminuição da sua função contrátil (15\%) ao exame ecocardiográfico, dados característicos de uma cardiomiopatia dilatada.

A observação de animais com amplitudes de onda $\mathrm{R}$ menores ou iguais a $2,5 \mathrm{mV}$ e com alterações ecocardiográficas significativas representou uma característica em nove dos 20 animais do presente estudo. A regressão linear demonstrou estatisticamente a baixa correlação entre a amplitude da onda $\mathrm{R}$ e a função ventricular $(\mathrm{r}=-0,36 / \mathrm{P}>0,05)$. Em quatro dos nove animais com amplitudes da onda $\mathrm{R}$ dentro dos padrões de normalidade, evidenciou-se, todavia, um aumento no tempo de duração do complexo QRS, superior a $0,04 \mathrm{~s}$, sugerindo, novamente, a sobrecarga ventricular esquerda. Deve-se à presença de uma correlação, estatisticamente significativa, entre 
a duração do complexo QRS e a função ventricular obtida pela ecocardiografia $(r=-0,58 / \mathrm{P}>0,05)$. Em outros dois animais, não foram observados aumentos da amplitude da onda $\mathrm{R}$, nem do tempo de duração do complexo QRS, mas um aumento pronunciado da amplitude da onda Q, na derivação II. Este parâmetro eletrocardiográfico pode ser considerado, um padrão de normalidade na raça Cocker Spaniel Inglês, mas a magnitude do seu aumento pode, ainda, representar em alguns animais, uma alteração eletrocardiográfica precoce para o diagnóstico da cardiomiopatia do Cocker Spaniel (GOODING et al.1986). Entretanto, não houve no presente estudo uma correlação estatística deste parâmetro eletrocardiográfico com a função ventricular $(r=-0,29 / P>0,05)$. Em outros três animais, as características morfológicas do complexo QRS não evidenciaram alterações significativas que sugerissem um padrão eletrocardiográfico de um animal com uma cardiomiopatia dilatada.

A fibrilação atrial é comumente observada em animais com cardiomiopatia dilatada das raças grandes e gigantes, porém os animais da raça Cocker Spaniel Inglês, quando acometidos por esta mesma doença miocárdica, apresentam como ritmo cardíaco predominante o sinusal. O presente estudo demonstrou que na maioria dos animais avaliados (12/ 20), as variações do ritmo sinusal (arritmia sinusal, bradicardia e taquicardia sinusal) representam o ritmo cardíaco mais comumente encontrado. Todavia, como anteriormente encontrado por THOMAS (1987), quando cães Cocker Spaniel Inglês possuem taquiarritmias atriais e ventriculares como ritmo predominante, observa-se uma evolução desfavorável do quadro clínico, particularmente naqueles com cardiomegalia severa, com um significativo aumento das câmaras atriais. De forma similar, observou-se a ocorrência de taquiarritmias atriais em oito animais, representando certamente aqueles com os maiores aumentos de cavidade atrial esquerda, tanto no exame ecocardiográfico como radiográfico, constituindo os animais com prognóstico mais reservado, face à gravidade dos achados clínicos. A arritmia ventricular foi um achado eletrocardiográfico infreqüente e, quando presente, fundamentalmente na forma de batimentos ventriculares isolados, exceção feita em dois casos clínicos, onde se observou um ritmo de fibrilação atrial alternado com batimentos ventriculares ectópicos num determinado período da evolução da doença.

A ocorrência de bradiarritmias, como bloqueio atrioventricular de segundo grau, em quatro dos doze animais com diagnóstico de cardiomiopatia dilatada, foi também identificada no presente estudo.
O bloqueio atrioventricular, assim como outras bradiarritmias, na cardiomiopatia da raça Cocker Spaniel Inglês representam uma importante diferenciação no padrão eletrocardiográfico em relação aos ritmos cardíacos observados nas formas clássicas de cardiomiopatia das raças grandes e gigantes, como no Doberman e o Boxer, visto que nestas raças, as taquiarritmias, indiscutivelmente constituem um achado comum.

As anormalidades ecocardiográficas, observadas no presente trabalho, foram evidenciadas pela perda da função contrátil miocárdica e pela dilatação ventricular esquerda, características da cardiomiopatia dilatada. A função ventricular nos animais afetados apresentou valores médios de $19 \%$, todavia, foram observados valores iguais ou inferiores a $12 \%$ em cinco animais, indicativos de severa disfunção contrátil ventricular. Não obstante, um dos casos clínicos foi um bom indicador da limitação e da baixa confiabilidade das alterações ecocardiográficas, observadas nos índices de ejeção, como fator único na determinação de um prognóstico, face ao bom controle terapêutico e a conseqüente prolongada sobrevida (cinco anos) em um animal que apresentava uma função ventricular de apenas $11 \%$. Em relação ao diâmetro diastólico final, a ecocardiografia bidimensional, ou de modo-M, identificou prontamente, o aumento da cavidade ventricular esquerda, revelando valores significativos, como os $6,0 \mathrm{~cm}$ de cavidade observado em um dos animais avaliados. A relação aorta/átrio esquerdo mostrou-se diminuída na totalidade dos animais avaliados, resultado da dilatação da cavidade atrial esquerda e, em menor grau, da redução do diâmetro da aorta em consequência do menor volume ejetado. Nos animais com dilatação ventricular grave, foi possível identificar a insuficiência da valva mitral, secundária à distensão do aparelho valvar (13 animais), agravando ainda mais o débito cardíaco. Foi ainda observado o espessamento dos folhetos valvulares da mitral, indicativo de uma endocardiose em quatro destes animais. Contudo, a severidade do quadro clínico e do sopro sistólico, detectado à auscultação, foi, fundamentalmente, resultados da regurgitação mitral, causada pela cardiomiopatia dilatada e não pela doença degenerativa da valva mitral.

\section{CONCLUSÕES}

$\mathrm{Na}$ análise dos resultados obtidos no presente trabalho, em relação aos aspectos clínicos, eletrocardiográficos, radiográficos e ecocardiográficos da cardiomiopatia da raça Cocker Spaniel Inglês, fundamentam-se as seguintes conclusões: 
os aspectos clínicos, eletrocardiográficos, radiográficos, e ecocardiográficos revelam características importantes que podem participar na diferenciação desta cardiomiopatia racial em relação à forma congestiva, idiopática, das raças grandes e gigantes;

não há predisposição sexual e a idade média dos animais acometidos é de sete anos, todavia podem ser observados casos clínicos com menos de um ano de idade;

a insuficiência cardíaca congestiva esquerda é a síndrome clínica predominante nos animais acometidos e, na presença de evoluções clínicas mais graves, é possível a ocorrência de insuficiência esquerda e direita (ascite);

a maioria dos animais apresenta uma evolução lenta e progressiva para uma insuficiência cardíaca congestiva esquerda, contudo observa-se uma rápida progressão para o óbito e a somátoria dos achados clínicos e de exames subsidiários revelaram um prognóstico pior; a cardiectasia, o desvio dorsal da traquéia e evidências de insuficiência cardíaca congestiva esquerda são achados radiográficos comuns na cardiomiopatia do Cocker Spaniel Inglês;

o ritmo cardíaco predominante é o sinusal com suas variações (arritmia, bradicardia e taquicardia), sendo que a amplitude da onda $\mathrm{R}$ acima de 2,5 $\mathrm{mV}$, o aparecimento de um "slurring" do segmento ST, e finalmente a maior profundidade da onda Q e da duração do complexo QRS constituem parâmetros adequados para a identificação de animais com cardiomiopatia dilatada nesta raça;

os achados ecocardiográficos característicos são o aumento das cavidades atrial e ventricular esquerda, a diminuição da força contrátil do miocárdio e a insuficiência mitral secundária à distenção do aparelho valvar;

com relação à evolução da doença parece haver dois padrões bem definidos. Um com rápida evolução para uma insuficiência cardíaca congestiva refratária aos tratamentos convencionais para o seu controle, tendo assim um prognóstico extremamente desfavorável, e um outro, que difere da evolução das cardiomiopatias das raças grandes e gigantes, onde a sobrevida extremamente longa com grande período assintomático é uma forma comumente identificada;

a observação de diferenças na evolução da doença podem sugerir que esta cardiomiopatia dilatada racial apresente hipoteticamente etiologias distintas da forma clássica.

\section{FONTES DE AQUISIÇÃO}

Nitrodisc-Biolab

Aminofilina inj-Mesquita

c Lasix-Hoechst

${ }^{d}$ Furosemide- I.Q.C. e Lasix-Hoechst

e Aldactone - Biolab

f Aminofilina-Sandoz

Teolong -knoll

${ }^{h}$ Renitec-Merck Sharp Dohme

Digoxina -Wellcome-Zeneca

\section{REFERÊNCIAS BIBLIOGRÁFICAS}

ATKINS, C.E.; SNYDER, P.S. Cardiomyopathy. In: ALLEN, D.G. Small animal medicine. Philadelphia : Lippincott, 1991. p.269-297.

BOON, J.A. Dilated cardiomyopathy. In: BOON, J.A. Manual of veterinary echocardiography. Baltimore : Williams \& Wilkins, 1998. p.320-333.

BUCHANAN, J.W. Causes and prevalence of cardiovascular disease. In: KIRK R.W.; BONAGURA J.D. (Ed). Current veterinary therapy XI. Philadelphia: Saunders, 1992. p.647655 .

FOX, P.R. Canine myocardial diseases. In: FOX, P.R. Textbook of canine and feline cardiology. New York : Churchill Livingstone, 1988. p.467-493.

GOODING, J.P.; ROBINSON, W.F.; MEWS, G.C. Echocardiographic characterization of dilatation cardiomiopathy in the English Cocker. American Journal of Veterinary Research, Chicago, v.47, n.9, p.1978-1982, 1986.

GOODWIN, J.F. Primary myocardial disease: spechum of cardiomyopathy and current classification. Singapore Medical Journal, Singapore, v.14, p.358, 1973.

HILL, B.L. Canine idiopathic congestive cardiomiopathy. The Compendium of Continuing Education for the Practicing Veterinarian, Princiton, v.3, p. 615-621, 1981 .

KITLLESON, M.D. Primary myocardial disease leading to chronic myocardial failure (dilated cardiomyopathy and related disease. In: KITLlESON, M.D.; KIENLE, R.D. Small animal cardiovascular medicine. St. Louis : Mosby, 1998. p. 319-346.

KRAMER, A.G.; KITTLESON, M.D.; FOX , P.R. Plasma taurine concentrations in normal dogs and in dogs with heart disease. Journal of Veterinary Internal Medicine, Philadelphia, v.9, n.4, p.253-258, 1995.

LARSSON, M.H.M.A. et al. Cocker Spaniel cardiomyopathy. Brazilian Journal Veterinary Research Animal Science, São Paulo, v.33, n.4, p.226-230, 1996.

SISSON, D.D., THOMAS, W.P. Myocardial diseases. In: ETTINGER, S.J.; FELDMAN, E.C. (Ed). Textbook of veterinary internal medicine. Philadelphia : Sauders, 1995. p.995-1032.

Ciência Rural, v.34, n.2, mar-abr, 2004. 
SPIEGEL, M.R. Estatística. São Paulo : Mac. Graw-Hill, 1972. 580p.

THOMAS, R.E. Congestive cardiac failure in young Cocker Spaniels (a form of cardiomiopathy ?): details of eight cases. Journal of Small
Animal Practice, London, GB, v.28, p.265-279, 1987 .

WARE, W.A.; BONAGURA, J.D. Canine myocardial disease. In: KIRK, R.W. Current veterinary therapy. 9.ed. Philadelphia : Saunders, 1986. p.370-380. 